\title{
Imaginarios sociales y su incidencia en la atención de las mujeres víctimas de violencia en el municipio de Sincelejo
}

\author{
Trabajo Resultados de Investigación \\ Margarita Irene Jaimes-Velásquez ${ }^{10}$
}

\section{Introducción}

En la actualidad el Estado colombiano cuenta con instituciones que permiten a la ciudadanía hacer valer sus derechos. Sin embargo, por razones que se discutirán en este escrito, las mujeres no tienen la atención instituida por la Ley 1257 de 2008 a la hora de reclamarlos. Por esta razón, este artículo presenta las problemáticas que tiene las mujeres en las Comisarías de Familia y los Centros de Atención de Víctimas de violencia sexual y violencia intrafamiliar de la Fiscalía en la ciudad de Sincelejo, con el personal al presentar reclamos cuando violan sus derechos. Esto, es debido a las representaciones sociales que tienen los comisarios y comisarias frente a las realidades de las mujeres víctimas de violencia de género. Por ende, el documento presenta los resultados obtenidos por medio de entrevistas al personal de dichas instituciones para reflejar las fallas que están respaldadas en sus imaginarios y, así, proponer soluciones para que la problemática no continúe afectando a la población de mujeres que exigen su derecho a una vida digna y libre de violencias.

${ }^{10}$ Doctoranda en Política y Gobierno de la Universidad Católica de Córdoba (Argentina), Maestra en Educación de los Derechos Humanos del Centro de Cooperación Regional para la Educación de Adultos en América Latina y el Caribe (México), Especialista en Derechos Humanos de la ESAP, Abogada. Docente Investigadora de la Facultad de Derecho y Ciencias Políticas de la Corporación Universitaria del Caribe-CECAR, Sincelejo, Colombia. Correo: margarita.jaimes@cecar.edu.co 
Para la compresión del tema, se abordará desde el cuerpo normativo vigente y los las elaboraciones subjetivas que estos, los funcionarios, hacen de las víctimas que acuden a sus despachos, a fin de describir como los imaginarios y estereotipos de género se constituyen en referentes interpretativos del quehacer institucional, develando el impacto de estas interpretaciones en el acceso a la justicia de las mujeres víctimas de violencia.

Para lograr este objetivo, los análisis tendrán en cuenta las obligaciones internacionales del Estado colombiano en materia de derechos humanos de las mujeres, qué medidas especiales para una adecuada atención a las mujeres víctimas de violencia de género; seguidamente, se expondrá el marco conceptual que guía este escrito, así como la metodología que permitió obtener los resultados expuestos, finalizando con las conclusiones a la luz de la Ley 1257 de 2008 y demás normas internacionales que tratan la materia.

\section{La protección legal de los derechos de las mujeres}

En materia de derechos humanos, el Estado colombiano tiene la obligación de adoptar todas las medidas necesarias a fin de garantizar, el goce, ejercicio y protección de los derechos humanos de toda la población que habita en él. Para ello, debe protegerlos a través de un régimen de derecho (Preámbulo Declaración Universal de los Derechos Humanos, 1948) para que, además tengan eficacia aun entre los particulares.

Existen dos obligaciones inmanentes al quehacer del Estado en esta materia: el deber de respetarlos, que le impone evitar que sus agentes o representantes los violen; mientras que la segunda, impone el deber de garantía (Art 2. Pacto de Derechos Civiles y Políticos, Art. 1 de la Convención Americana de Derechos Humanos) Lo anterior, se materializa en la Constitución Política (1991) cuando reza que, las instituciones de la Republica están instituidas "para proteger a todas las personas residentes en Colombia, en su vida, honra, bienes, creencias, y demás derechos y libertades, y para asegurar el cumplimiento de los deberes sociales del Estado y de los particulares" (Art.2).

Ahora, por Bloque de Constitucionalidad se han incorporado otras normas del derecho internacional de los Derechos Humanos que imponen obligaciones específicas en materia de protección de los derechos de las mujeres. En ese sentido Convención Interamericana para prevenir, sancionar 
y erradicar la violencia contra la mujer "Convención De Belem Do Para" (1994), instituye que los Estados deberán realizar medidas apropiadas para modificar los patrones socioculturales y las prácticas históricas basadas en estereotipos o en la creencia de superioridad de uno de los sexos (Art.5).

De otro lado, la misma, impone a los Estados la obligación de: i.) Abstenerse de cualquier acción o práctica de violencia contra la mujer y velar por que las autoridades, sus funcionarios, personal y agentes e instituciones se comporten de conformidad con esta obligación; ii.) Actuar con la debida diligencia para prevenir, investigar y sancionar la violencia contra la mujer (Art. 7) Además insta a que se modifiquen los patrones socioculturales de conducta de hombres y mujeres, a fin de evitar prejuicios y costumbres basados en estereotipos que legitimen la violencia contra la mujer, para ello, debe fomentar la capacitación de los funcionarios encargados de la aplicación de la ley (Art. 8). En ese sentido, la Convención para eliminar todas las formas de discriminación contra la mujer (1979) estableció que la protección jurídica de las mujeres debe estar sustentada en la igualdad entre hombres y mujeres.

Como se observa, las obligaciones del Estado colombiano no quedan expresadas en términos de abstención, sino que apuntan a la transformación de los patrones socioculturales que perpetuán la discriminación y la violencia contra las mujeres, específicamente, entre el grupo de funcionarios públicos que deben atender las situaciones que afectan los derechos de las mujeres, ya que, esos patrones estereotipados se constituyen en barreras de acceso a la justicia haciendo ineficaces las normas que buscan prevenir y proteger los derechos de las mujeres.

En ese orden de ideas, el 4 de diciembre de 2008, nace a la vida jurídica la Ley 1257 que tiene por objeto adoptar normas que permitan a las mujeres vivir una vida libre de violencia, desarrolla conceptualmente la violencia, reconoce los derechos de las mujeres víctimas e impone tareas claras, precisas y exigibles a los operadores de justicia en materia de investigación y sanción penal, así como medidas para la protección de las víctimas y sus familiares ante las instancias administrativas y judiciales.

De lo anterior se colige que el restablecimiento de los derechos y el acceso al derecho a la justicia es una responsabilidad ineludible para el 
Estado, que debe ser cumplida cabalmente por sus funcionarios, quienes, deben actuar con la debida diligencia (Convención Belem do Pará), evitando la revictimización y la convalidación de estereotipos de género que incrementen la vulnerabilidad de la víctima y comprometan la responsabilidad estatal.

En ese sentido algunos estudios se han centrado en la eficacia de las medidas de atención (Jaimes y Escobar, 2014) otros en las medidas de protección (Corporación Humanas, 2016) y otros en las prácticas y discursos legitimadores de la violencia contra las mujeres (Gómez, Murad y Calderón, 2013) Sin embargo, en materia de imaginarios sociales de los funcionarios y funcionarias que atienden casos de violencia contra las mujeres y sus implicaciones en el acceso a la justicia pocos son los avances investigativos en este país. No obstante en el ámbito internacional, la Comisión Interamericana de Derechos Humanos en el informe de Informe Acceso a la justicia para las mujeres víctimas de la violencia en las Américas (2007):

La persistencia de patrones y comportamientos socioculturales discriminatorios que obran en detrimento de las mujeres, que impiden y obstaculizan la implementación del marco jurídico existente y la sanción efectiva de los actos de violencia, a pesar que este desafío ha sido identificado como prioritario por los Estados americanos (párr. 8).

Para reafirmar esto, la honorable Corte Constitucional ha dicho que las mujeres son sujetos de especial protección constitucional, por lo que toda actuación del Estado Social de Derecho se debe sustentar en la dignidad humana (Sentencia C. 804 de 2006) recordando además que las situaciones de inclusión y exclusión también se evidencian en el lenguaje jurídico convocando a que la visibilización de las mujeres y sus realidades a fin de armonizar con lo dispuesto en los tratados internacionales aprobados por Colombia. En la sentencia T-027 de 2017 reitera la condición de especial protección de las mujeres, instando a evitar los estereotipos de género, recordando que una modalidad de violencia contra las mujeres ocurre cuando hay permisividad o tolerancia de parte del Estado (Sentencia C-335 de 2013). Ha dicho que el funcionario se puede equivocar cuando valora caprichosa y arbitrariamente las pruebas o situaciones presentadas, por lo que reitera que, el Estado debe asumir un rol activo en la eliminación de los estereotipos de género (Sentencia T 967 de 2014). 


\section{Imaginarios}

Este concepto ha sido abordado desde distintas disciplinas, Castoriades (1993) reflexiona sobre la sociedad y sus modos de imponerse mediante la formación de la materia prima, el ser humano. Para él, las significaciones imaginarias no corresponden a la realidad o la racionalidad, ya que solo existen estando instituidas y reproducidas por una colectividad. Expone que toda significación imaginaria se correlaciona con otro sinnúmero de imaginarios que se manifiestan a través de la lengua.

Según García Calcini (2007), los imaginarios son elaboraciones subjetivas de lo que existe, elaboraciones simbólicas de la realidad que se explica por la imposibilidad de un conocimiento universal, totalizador y permeado por narrativas diversas. En su texto la imaginación simbólica (Durand, 1968) afirma que el símbolo hace parte del signo que busca economizar o remitir a un significado cuando es imposible representar algo y por tanto, lo imaginario llena los vacíos de aquello que no tiene explicaciones o que no sabemos. Se constituye en un substrato ideológico producido colectivamente. De ahí que se constituye en una forma de mirar, pensar y de estar en el mundo aunque matizado por la historia de vida de cada individuo (Durkheim, citado por Padilla, 2006). Explican otros autores que para entender su significado es necesario entender la historia de su producción (Luckmann, 1968) Así las cosas, se entiende que los imaginarios sociales crean un orden social sobre el cuerpo colectivo que se trasmite y perpetúa en la conciencia de la colectividad. Esos esquemas organizadores plantean códigos sociales que encapsulan a las mujeres en unos órdenes específicos.

\section{Estereotipos y roles de género}

El sexo es un producto de la biología humana, no cambia, permanece inmutable a través del tiempo sin importar el tiempo o el contexto. Contrario sensu, el género, como lo menciona Marcela Lagarde (1993), es construido por diversos factores, políticos, económicos y culturales, haciéndolo relativo a una comunidad. Por tanto, no es biológico, sino un asunto social que muta y evoluciona con la comunidad. 
Esos roles construidos a partir de las relaciones culturales y políticas, establecen cargas a las mujeres en el ámbito estrictamente doméstico. Autoras como Françoise Collin (1994) cuando habla del espacio doméstico, explica que este, es un espacio comportamental que establece formas de relacionamiento diferenciada, ya que la costumbre rige sus relaciones cotidianas. Esta división de roles encomendados a cada sexo comienza estableciendo un sistema que asigna las labores a desempeñar a partir de lo sexual (Barrios, 2008). Como dice Laura Guzmán (s.f) son "las expectativas sociales creadas en torno al comportamiento femenino y masculino" (p.1).

Como se lee, los roles de género otorgan privilegios a lo masculino, valiéndose en sus instituciones de reproducción: la familia, la escuela y la cultura. Los roles de género se sustentan en el sexismo y la estructura androcéntrica que explica y sustenta la violencia contra las mujeres (Facio, 2002). Estos roles son producto de su socialización histórica de modo que se han naturalizado invisibilizando la inequidad (Eagly, 1987, citada por Godoy y Mladinic, 2009).

De lo anterior devienen los estereotipos de género que están íntimamente ligados a los prejuicios. Se entiende por estereotipo, según Mackie (1973, citado por González, 1993) aquellas creencias que caracterizan un grupo social y sobre las que hay un acuerdo básico que en ocasiones, anula la identidad de la persona. Los estereotipos persisten a través del tiempo (Levine, 1972) y son mantenidos inalterables por el grupo social, siempre y cuando, el grupo estereotipador no necesite cambiarlo (González, 1999).

\section{Metodología}

Para este ejercicio es descriptivo desde un enfoque de investigación cualitativa (Taylor y Bogdan, 2004) debido a que sirvió para identificar y exponer los imaginarios sociales de los equipos humanos que prestan sus servicios en la atención a las mujeres víctimas de violencia basada en género en las comisarías de familia y la Fiscalía de la ciudad de Sincelejo. La población objeto de estudio fueron las funcionarias y funcionarios de las dos comisarías de familia de la ciudad de Sincelejo, los fiscales de la Unidad de Reacción Inmediata, el Centro para la Atención Integral a las Víctimas de Violencia Sexual y el Centro de Atención a la Víctima de Violencia Intrafamiliar de la Fiscalía General de Nación en Sincelejo. 
Los instrumentos aplicados fueron entrevistas semi-estructuradas (Díaz-Bravo, Torruco-García, Martínez-Hernández y Varela-Ruiz, 2013) al 100\% del personal de las comisarías de familia de las dos comisarías de familia existentes en la Ciudad de Sincelejo, entrevistas abiertas y a profundidad al comisario primero y comisaria segunda, y Fiscales URI, CAIVAS, CAVIF y Fiscales locales de la ciudad de Sincelejo, la observación en las salas de espera y los diarios de campo llevados por cada integrante del semillero que dio cuenta de las impresiones inmediatas de cada actividad realizada.

Las entrevistas fueron trianguladas (Flick, 2012) con los diarios de campo. La triangulación no se realizó para verificar si el funcionario tiene la razón, sino, para identificar diferentes puntos de vista y si estos responden a las obligaciones del Estado frente a la necesidad de las mujeres de acceder efectivamente a la justicia.

\section{Resultados de las percepciones e imaginarios}

En la investigación denominada el Acceso a la Justicia como derecho fundamental en la atención de la violencia de género — en adelante, VGse indagó sobre los imaginarios y estereotipos que pueden incidir en la atención que se ofrece a las mujeres víctimas de VG en las unidades de la Fiscalía y en las Comisarias de Familia de la ciudad de Sincelejo.

En primera instancia se encontró que categorizan en una escala de menor a mayor (1 a 5) como de suma importancia los derechos de los niños, niñas y adolescentes con un promedio de 4,83 , y en segundo lugar $(4,67)$ los derechos de las mujeres, quedando en el mismo promedio el concepto de la reconciliación y la condición de victimización con un 4,50, en quinto lugar de importancia quedó la sanción con un promedio de 4,33, mientras que el tema de acceso a la justicia aparece con el menor promedio con un 4,17 , junto al concepto de la unidad familiar. Sin embargo todos los temas son considerados relevantes, toda vez que en promedio están por encima de 4,0 .

En cuanto al lenguaje incluyente como una forma de reafirmar los derechos de las mujeres, el 66,7\% de los funcionarios y funcionarias encuestadas lo consideró importante, mientras que el 16,7\% afirmó que no es 
importante E1 83,3\% cree que en Colombia las mujeres tienen una mayor protección que los hombres y el 100\% considera que en algunos casos las mujeres provocan la violencia de la que son víctimas. En ese orden de ideas, el 83,3\% expresa que existen actitudes de las mujeres que pueden provocar la violencia sexual; por otra parte, el $16,7 \%$ piensa que la reputación de la mujer es un elemento determinante para ser reconocida como víctima de abuso y/o violencia sexual.

\section{Imaginarios culturales y sociales frente a la violencia de género}

Cuando el personal encargado de proteger los derechos de las mujeres considera de poca importancia los derechos de las mujeres anteponiendo los derechos de los hijos, se revictimiza a la afectada imponiéndole la carga moral de ser madres ante todo. Este tipo de análisis sesgados, explica por qué las acciones de atención local, se concentran en medidas reactivas una vez ha acontecido el hecho dañino y, no en las medidas preventivas y protectoras de largo alcance que señala la Ley 1257 de 2008.

Desde esa postura, es posible entender por qué el acceso a la justicia quedó al final de la Tabla . Priman las miradas estereotipadas ligadas al rol familiar dejando de lado el derecho a la justicia de las mujeres víctimas de violencia de género. Este sistema de valoración permea la compresión del entorno en que ocurre la violencia restándole importancia a los daños que sufre la mujer incrementando la discriminación y la impunidad.

Cuando la sanción no es importante, no se inician los procedimientos necesarios para determinar la culpabilidad del agresor, como tampoco las acciones para proteger a las mujeres, de modo que es posible invitar a la víctima a reconciliarse con su agresor como quedó evidenciado en los resultados.

De modo que, las comisarías y unidades especializadas de Fiscalía, incumplen el Artículo 4 de la Ley 1257 de 2008 al no interpretar y aplicar los principios constitucionales, los tratados internacionales en materia de derechos humanos ratificados por Colombia, y, en especial, las Convenciones Belem do Pará y la CEDAW; ni tener en cuenta la abundante jurisprudencia constitucional cuando expone que la justicia es un pilar importante del estado social de derecho, que no puede quedar reducido a un mero con- 
cepto legal y teórico, sino que exige acciones inmediatas y con resultados en favor de la víctima (Sentencia C-297 de 2016).

Para evitar esto, se precisa que el proceso de reclutamiento de los funcionarios y funcionarias incluya pruebas o entrevistas que permitan identificar cuáles son los estereotipos o los imaginarios de cada aspirante en relación a los roles de género. Lo anterior es sumamente importante, en tanto, estos imaginarios intervienen negativamente en la competencia e idoneidad de la función pública.

El lenguaje es una construcción social que refleja cómo está pensada la sociedad, en ese sentido, un $90 \%$ de los funcionarios y funcionarias respondió que era importante el lenguaje incluyente, sin embargo, no lo usan, como tampoco lo han interiorizado. Al revisar todas las respuestas a la luz del lenguaje incluyente se encuentra una relación directa entre lo que se piensa, lo que se expresa y lo que hacen. De hecho, algunos funcionarios consideran sin importancia el lenguaje incluyente en su labor, obviando que la Corte Constitucional ha dicho que:

Siendo el lenguaje, como lo es, uno de los principales instrumentos de comunicación y reflejando — como refleja — los hábitos, concepciones y valores imperantes en un medio social determinado, las situaciones de inclusión o exclusión también se proyectan en el lenguaje jurídico. Precisamente uno de los fines de las definiciones legales consiste en fijar fronteras y, en tal sentido, en determinar quiénes se encuentran dentro de los límites establecidos por las definiciones y quiénes permanecen por fuera. Los criterios que se utilizan para marcar la inclusión o la exclusión reflejan el contexto valorativo e ideológico en que ellos se adoptan. "el lenguaje no solo refleja y comunica los hábitos y valores de una determinada cultura sino que conforma y fija esos hábitos y valores (...) (Sentencia C-804 de 2006).

¿A qué se debe este incumplimiento de los estándares legales, si todos son profesionales del derecho? La respuesta no es simple; sin embargo, un factor importante, es la lectura estereotipada de la realidad que invisibiliza la discriminación y la violencia contra las mujeres. Los imaginarios sociales que aceptan y conceden privilegios a los hombres sobre los cuerpos de las mujeres, deben ser superados por aquellos que ostentan la calidad de protectores de los derechos de las mujeres en los términos de la Ley 1257 de 2008. 
El cuerpo de funcionarios y funcionarias consultadas consideran que en Colombia, las mujeres tienen mayor protección que los hombres. Es prudente advertir que no fue posible hallar respuestas que ampliaran las razones que respaldan tal aseveración. Se puede suponer que las violencias contra las mujeres, no son interpretadas en contextos de relaciones de poder, en las que las mujeres son subyugadas, sino que son percibidas por estos funcionarios, como conflictos entre pares con la misma capacidad para ejercerlo, por ello, susceptible de conciliación. Esa lectura poco ajustada a la realidad afianza la desigualdad, incrementa la vulnerabilidad de las víctimas y se constituye en una barrera de acceso a la justicia para ellas.

Es diciente que el 83,3\% del personal entrevistado no pueda explicar las razones legales, políticas y éticas de la protección a las mujeres, máxime cuando se presumen expertos en la materia. Ello tiene respuesta en la subjetividad del funcionario o funcionaria en quien subsisten creencias e imaginarios sobre lo que es ser mujer u hombre en la sociedad sucreña.

Mucho más preocupante resulta que el $66,7 \%$ cree que ciertas actitudes de las mujeres provocan la violencia sexual, es decir, trasladan la responsabilidad a la víctima, justificando la violencia y a su perpetrador. Es contrario al derecho que se les exija a las víctimas de violencia sexual un tipo de conducta para reconocerles su condición de víctimas. De hecho, tanto la Ley 1257, como la Convención Belem de Pará y la jurisprudencia Constitucional han reiterado que la vida sexual de la víctima no puede ser un elemento de valoración al momento de atender los casos de violencia sexual contra las mujeres (Art 8). Cuando esto no es tenido en cuenta, se amplifica la vulnerabilidad de las victimas al despojarlas de sus derechos a la justicia, la verdad y el restablecimiento de sus derechos (Sentencia C-297 de 2016).

Los imaginarios sociales que sostienen que las mujeres deben ser santas para ser respetadas y reconocidas en los estrados judiciales o administrativos inciden en la perpetuación de la violencia contra las mujeres, quienes además, al sentirse desprotegidas y estigmatizadas pueden preferir no denunciar. De otro lado, este tipo de imaginarios afectan la eficiencia y la eficacia con que se debe realizar la investigación penal generando altos niveles de impunidad. 
Las valoraciones sustentadas en los imaginarios culturales o los estereotipos de género en sede judicial o administrativa se convierten en obstáculos para el acceso a la justicia, reproduciendo la violencia estructural que atenta contra la dignidad humana de las mujeres.

\section{Conclusiones}

Los imaginarios culturales arraigados en la conciencia de los funcionarios y funcionarias hacen ineficaces los contenidos normativos, en tanto, desdibujan la objetividad con que se deben interpretar y aplicar las normas.

Los imaginarios afectan los diagnósticos situacionales que debe realizar cada funcionario o funcionaria en su labor, ya que, se hacen valoraciones situadas en los roles de género de modo que terminan justificando, naturalizando o minimizando los actos violentos contra las mujeres trasladando la culpa a ellas y no en el agresor.

Las miradas estereotipadas de las relaciones entre hombres y mujeres, afecta gravemente la dignidad humana de las mujeres, re victimizándolas y amplificando la violencia estructural y perpetuando desde el Estado la discriminación y subyugación de estas en relación a los hombres.

La debida diligencia en las actuaciones judiciales o administrativas, no puede ser una exigencia en abstracto, sino que por el contrario, debe propender por la transformación de la cultura, la legislatura y la justicia, a fin de evitar la impunidad y poner fin a la violencia contra las mujeres. Por ello, se hace necesario que, tanto funcionarias como funcionarios de las Comisarias y de la Fiscalía, transformen su quehacer desde nuevas apuestas incluyentes encaminadas a derribar los imaginarios que sustentan la inferioridad de las mujeres en relación a los hombres a fin de hacer objetivas sus actuaciones y sus decisiones.

Las estipulaciones de la Ley 1257 de 2008, son criterios fundamentales para analizar, sin prejuicios, los casos de violencia contra las mujeres. Ignorarlos no solo constituye una violación a la normativa específica, sino también, a los fines esenciales del Estado colombiano. 


\section{Referencias bibliográficas}

Barrios David. (2008) Resignificar lo masculino. Editorial Vila Editores.

Beauvoir Simone. (1949) El segundo Sexo. Nueva York. http://users.dsic. upv.es/ pperis/El\%20segundo\%20sexo.pdf

Berger, Peter y Luckmann, Thomas. (1968). La construcción social de la realidad. Amorrortu Editores. Buenos Aires.

Castoriadis, Cornelius (1975) La institución imaginaria de la Sociedad. Tusquets Editores, Buenos Aires.

Comisión Interamericana de Derechos Humanos (2007) Informe Acceso a la justicia para las mujeres víctimas de la violencia en las Américas, recuperado de https://www.cidh.oas.org/women/acceso07/cap1.htmES

Constitución Política de Colombia (1991). Artículo 2.

Congreso de la Republica de Colombia. Ley 1257 de 2008. Por la cual se dictan normas de sensibilización y discriminación contra las mujeres, se reforman los Códigos penal, de procedimiento penal, la Ley 294 de 1996 y se dictan otras disposiciones.

Corte Constitucional, Sentencia C-804 de 2006. (MP. Humberto Antonio Sierra Porto septiembre 27 de 2006).

Corte Constitucional, Sentencia T-878 de 2014. (MP. Jorge Iván Palacio, 18 Noviembre de 2014).

Corte Constitucional, Sentencia T-967 de 2014 (MP. Gloria Ortiz Delgado, 15 de diciembre de 2014).

Corte Constitucional, Sentencia C-297 de 2016 (MP. Gloria Ortiz Delgado, 8 de junio de 2016).

Corte Constitucional, Sentencia T-027 de 2017 (MP. Jorge Ignacio Pretelt Chaljub, 13 de junio de 2013). 
Díaz-Bravo, Laura, Torruco-García, Uri, Martínez-Hernández, Mildred, y Varela-Ruiz, Margarita. (2013). La entrevista, recurso flexible y dinámico. Investigación en educación médica, 2(7), 162-167. Recuperado en 11 de marzo de 2018, de http://www.scielo.org.mx/scielo.php?script=sci_Arttextypid=S2007-50572013000300009ylng=esytlng=es

Durand, Gilbert. (2000). La imaginación simbólica. Amorrortu editores. Buenos Aires.

Facio, Alda (2002) Con los lentes de Género se ve otro derecho. El otro Derecho (28), 85-102.

Flick, Uwe. (2012). Introducción a la investigación cualitativa. Editorial Paideia. Madrid.

García, Néstor (2007) Que son los imaginarios y cómo actúan en la ciudad. Revista eure, 33(99), 89-99. Santiago de Chile.

García, Juan (2000) Diarios de campo. Volumen 31. Editorial Centro de Investigaciones Sociológicas.

Godoy, Lorena, y Mladinic, Antonio. (2009). Estereotipos y Roles de Género en la Evaluación Laboral y Personal de Hombres y Mujeres en Cargos de Dirección. Psykhe (Santiago), 18(2), 51-64. https://dx.doi. org/10.4067/S0718-22282009000200004

Gómez, C; Murad, R y Calderón, M. (2013) Historias de violencia, roles, prácticas y discursos legitimadores. Violencia contra las mujeres en Colombia 2000-2010. Recuperado de: http://www.profamilia.org.co/ docs/estudios/imagenes/7\%20-\%20VIOLENCIA\%20CONTRA\%20 LAS\%20MUJERES\%20EN\%20COLOMBIA.pdf

González, Blanca (1999), Los estereotipos como factor de socialización en el género Comunicar. Sevilla, España.

Guzmán, L (s.f). Roles sexuales, Roles de género y poder. Recuperado de http://www.ts.ucr.ac.cr/binarios/docente/pd-000124.pdf 
Jaimes, Margarita y Escobar, Justa. (2014). Violencia de Género e Instituciones. Atención y Tratamiento de las Mujeres en la Ciudad de Sincelejo en el Marco de la Ley 1257 de 2008. Editorial Tecnológico Comfenalco, pp.122-137.

Lagarde, Marcela. (1996) “El género”, fragmento literal: 'La perspectiva de género', en Género y feminismo. Desarrollo humano y democracia, Ed. horas y HORAS, España.

LeVine, Robert y Campbell, Donald (1972): Ethnocentrism: Theories of Conflict, Ethnic Attitudes and Group Behavior. Nueva York, Wiley.

Organización de Estados Americanos O.E.A. (1994). Convención Interamericana para Prevenir, Sancionar y Erradicar la Violencia Contra la Mujer.

Organización de Estados Americanos O.E.A. (1969). Convención Americana de Derechos Humanos.

Organización de las Naciones Unidas ONU (1976) Pacto Internacional de Derechos Civiles y Políticos.

Organización de las Naciones Unidas ONU (1979). Convención sobre la Eliminación de todas las Formas de Discriminación contra la Mujer.

Organización de las Naciones Unidas ONU (1948). Declaración Universal de los Derechos Humanos.

Padilla, M. (2006). Emile Durkheim estructura social y subjetividad. Editorial Universidad Autónoma del Estado de Hidalgo. Pachuca.

Taylor, S. y Bogdan, R. (2004). Introducción a los métodos cualitativos de la investigación. Recuperado de: https://asodea.files.wordpress.com/2009/09/ taylor-s-j-bogdan-r-metodologia-cualitativa.pdf 